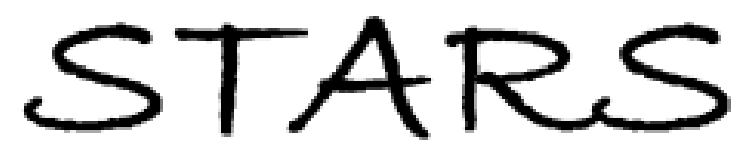

University of Central Florida

STARS

Faculty Bibliography 2000s

Faculty Bibliography

$1-1-2004$

\title{
Temperature effect on liquid crystal refractive indices
}

Jun Li

University of Central Florida

Sebastian Gauza

University of Central Florida

Shin-Tson Wu

University of Central Florida

Find similar works at: https://stars.library.ucf.edu/facultybib2000

University of Central Florida Libraries http://library.ucf.edu

This Article is brought to you for free and open access by the Faculty Bibliography at STARS. It has been accepted for inclusion in Faculty Bibliography 2000 s by an authorized administrator of STARS. For more information, please contact STARS@ucf.edu.

\section{Recommended Citation}

$\mathrm{Li}$, Jun; Gauza, Sebastian; and Wu, Shin-Tson, "Temperature effect on liquid crystal refractive indices" (2004). Faculty Bibliography 2000s. 4536.

https://stars.library.ucf.edu/facultybib2000/4536

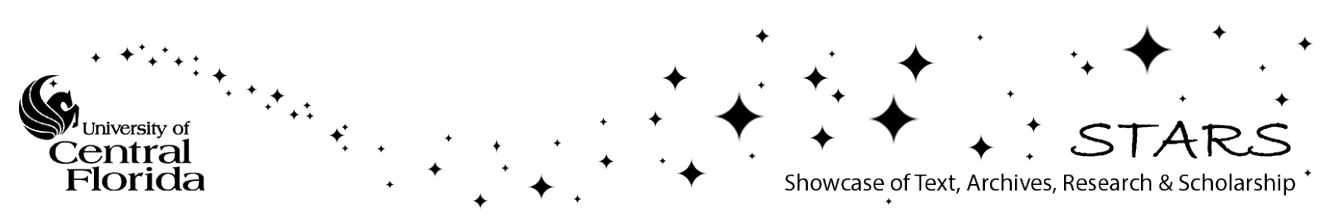




\section{Temperature effect on liquid crystal refractive indices}

Cite as: Journal of Applied Physics 96, 19 (2004); https://doi.org/10.1063/1.1757034

Submitted: 04 March 2004 . Accepted: 07 April 2004 . Published Online: 17 June 2004

Jun Li, Sebastian Gauza, and Shin-Tson Wu

\section{ARTICLES YOU MAY BE INTERESTED IN}

Infrared refractive indices of liquid crystals

Journal of Applied Physics 97, 073501 (2005); https://doi.org/10.1063/1.1877815

Extended Cauchy equations for the refractive indices of liquid crystals

Journal of Applied Physics 95, 896 (2004); https://doi.org/10.1063/1.1635971

Temperature-dependent optical constants and birefringence of nematic liquid crystal 5CB in the terahertz frequency range

Journal of Applied Physics 103, 093523 (2008); https://doi.org/10.1063/1.2913347

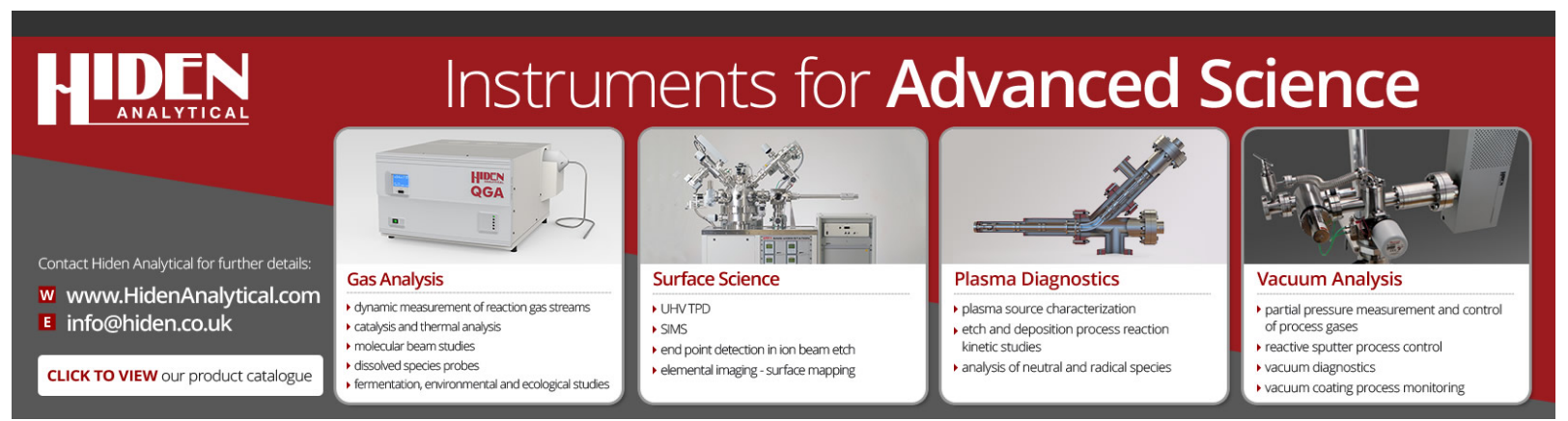




\title{
Temperature effect on liquid crystal refractive indices
}

\author{
Jun Li, Sebastian Gauza, and Shin-Tson Wua) \\ College of Optics and Photonics, University of Central Florida, Orlando, Florida 32816
}

(Received 4 March 2004; accepted 7 April 2004)

\begin{abstract}
A model describing the temperature effect of liquid crystal (LC) refractive indices is derived and confirmed by experiment. Two single LC compounds $(5 \mathrm{CB}, 5 \mathrm{PCH})$ and two mixtures (MLC-6241-000 and UCF-35) with different birefringence values were used to validate the model. This model fits all the experimental data well. For a low-birefringence LC mixture, if the operating temperature is far below its clearing point, the temperature-dependent refractive indices can be approximated as a parabolic form. This prediction is also experimentally validated. In addition, a linear relationship between $\left\langle n^{2}\right\rangle$ and temperature is found. (C) 2004 American Institute of Physics.
\end{abstract}

[DOI: $10.1063 / 1.1757034]$

\section{INTRODUCTION}

Liquid crystal (LC) has been used extensively for directview and projection displays, tunable photonics, and nonlinear optics. ${ }^{1-4}$ Most of the LC devices use the electric-, thermal-, or optical-field-induced refractive index change to modulate light. LC refractive indices are determined mainly by the molecular structure, wavelength, and temperature. Several models, ${ }^{5-10}$ such as the single-band model, ${ }^{5}$ threeband model ${ }^{8}$ and extended Cauchy model, ${ }^{10}$ have been developed to describe the wavelength-dependent $\mathrm{LC}$ refractive indices. The three-band model is particularly useful for understanding the origins of the refractive indices of single LC compounds, while the extended Cauchy model is more appropriate for LC mixtures. As the wavelength increases, both $n_{e}$ (refractive index for the extraordinary ray) and $n_{o}$ (refractive index for the ordinary ray) decrease, and then gradually saturate in the near-infrared region. ${ }^{5}$

Temperature also plays an important role in affecting the LC refractive indices. As the temperature increases, $n_{e}$ behaves differently from $n_{o}$. The derivative of $n_{e}$ (i.e., $\left.\partial n_{e} / \partial T\right)$ is always negative. However, $\partial n_{o} / \partial T$ changes from negative to positive as the temperature exceeds the crossover temperature. ${ }^{11}$ For elevated temperature operation of a LC device, temperature-dependent refractive indices need to be modeled accurately. Some semi-empirical models ${ }^{12-14}$ have been developed for describing the temperature effect on the LC refractive indices. However, a comprehensive model would be much more desirable.

In this article, we derive a model for describing the temperature-dependent LC refractive indices based on the Vuks equation. ${ }^{15}$ For low-birefringence thin-film-transistor (TFT) LC materials with a high clearing point $\left(>100^{\circ} \mathrm{C}\right)$, the temperature-dependent $\mathrm{LC}$ refractive indices have a parabolic form. In Sec. II, we show the derivation processes for the temperature-dependent refractive indices. In Sec. III, the experimental method for measuring the refractive indices is briefly described. In Sec. IV, we validate the models by fitting the experimental data of four LC compounds and mix-

\footnotetext{
a)Electronic mail: swu@mail.ucf.edu
}

tures with different birefringences. Excellent agreement between theory and experiment is obtained. We use the experimental data of MLC-6241-000 ( $\Delta n \sim 0.085, T_{c}$ $\sim 100^{\circ} \mathrm{C}$ ) to validate the parabolic model. This model fits the experimental data very well when the temperature is far from the clearing point. Finally, a linear relationship between $\left\langle n^{2}\right\rangle$ and temperature is discussed.

\section{THEORY}

The classical Clausius-Mossotti equation ${ }^{16}$ correlates the dielectric constant $(\varepsilon)$ of an isotropic medium with its molecular polarizability $(\alpha)$ at low frequencies. Replacing $\varepsilon=n^{2}$, the Lorentz-Lorenz equation ${ }^{16}$ correlates the refractive index of an isotropic medium with molecular polarizability in the optical frequencies. In principle, these two equations are derived for isotropic media and are not suitable for anisotropic liquid crystals in which dielectric constants $\left(\varepsilon_{\|}, \varepsilon_{\perp}\right)$ and molecular polarizabilities $\left(\alpha_{\|}, \alpha_{\perp}\right)$ are all anisotropic in the nematic phase.

Vuks made a bold assumption that the internal field in a $\mathrm{LC}$ is the same in all directions and gave a semi-empirical equation correlating the refractive indices with the molecular polarizabilities for anisotropic materials: ${ }^{15}$

$$
\frac{n_{e, o}^{2}-1}{\left\langle n^{2}\right\rangle+2}=\frac{4 \pi}{3} N \alpha_{e, o} .
$$

In Eq. (1), $n_{e}$ and $n_{o}$ are refractive indices for the extraordinary ray and ordinary ray, respectively, $N$ is the number of molecules per unit volume, $\alpha_{e, o}$ is the molecular polarizability, and $\left\langle n^{2}\right\rangle$ is defined as

$$
\left\langle n^{2}\right\rangle=\left(n_{e}^{2}+2 n_{o}^{2}\right) / 3 .
$$

In Eq. (1), $n_{e}$ and $n_{o}$ are coupled together so that the relationship between the refractive indices and the corresponding molecular polarizabilities is not clear. To reveal this relationship, we should decouple $n_{e}$ from $n_{o}$ by solving Eq. (1). Substituting Eq. (2) to Eq. (1) and separating $n_{e}$ and $n_{o}$, we obtain $^{10}$ 


$$
\begin{aligned}
& n_{e}=\left(1+\frac{4 \pi N \alpha_{e}}{1-\frac{4}{3} \pi N\langle\alpha\rangle}\right)^{1 / 2}, \\
& n_{o}=\left(1+\frac{4 \pi N \alpha_{o}}{1-\frac{4}{3} \pi N\langle\alpha\rangle}\right)^{1 / 2},
\end{aligned}
$$

where $\langle\alpha\rangle$ is the average polarizability of the LC molecule and is defined as ${ }^{17}$

$$
\langle\alpha\rangle=\frac{\alpha_{e}+2 \alpha_{o}}{3} .
$$

When $N\langle\alpha\rangle$ is small, Eqs. (3) and (4) can be expanded into a power series. Retaining the first two terms, we derive $\mathrm{e}^{10}$

$$
\begin{aligned}
& n_{e} \approx \frac{3 \sqrt{2}}{4}+\frac{\sqrt{2} \pi N\langle\alpha\rangle}{1-\frac{4}{3} \pi N\langle\alpha\rangle}+\frac{\frac{2 \sqrt{2}}{3} \pi N S\left(\gamma_{e}-\gamma_{o}\right)}{1-\frac{4}{3} \pi N\langle\alpha\rangle}, \\
& n_{o} \approx \frac{3 \sqrt{2}}{4}+\frac{\sqrt{2} \pi N\langle\alpha\rangle}{1-\frac{4}{3} \pi N\langle\alpha\rangle}-\frac{\frac{\sqrt{2}}{3} \pi N S\left(\gamma_{e}-\gamma_{o}\right)}{1-\frac{4}{3} \pi N\langle\alpha\rangle} .
\end{aligned}
$$

Birefringence of a LC material is defined as $\Delta n=n_{e}-n_{o}$. Subtracting Eq. (7) from Eq. (6), we obtain

$$
\Delta n \approx \frac{\sqrt{2} \pi N S\left(\gamma_{e}-\gamma_{o}\right)}{1-\frac{4}{3} \pi N\langle\alpha\rangle} .
$$

The average refractive index of a LC is defined as

$$
\langle n\rangle=\frac{n_{e}+2 n_{o}}{3} .
$$

Substituting Eqs. (6) and (7) into Eq. (9), we derive

$$
\langle n\rangle \approx \frac{3 \sqrt{2}}{4}+\frac{\sqrt{2} \pi N\langle\alpha\rangle}{1-\frac{4}{3} \pi N\langle\alpha\rangle} .
$$

Substituting Eqs. (8) and (10) back to Eqs. (6) and (7), the refractive indices have the following simple expressions:

$$
\begin{aligned}
& n_{e}=\langle n\rangle+\frac{2}{3} \Delta n, \\
& n_{o}=\langle n\rangle-\frac{1}{3} \Delta n .
\end{aligned}
$$

In theory, both $n_{e}$ and $n_{o}$ are functions of wavelength and temperature. The wavelength effect has been addressed extensively. Here, we focus on the temperature effect. According to our experimental data and fitting results, the average refractive index $\langle n\rangle$ decreases linearly as the temperature increases: ${ }^{11}$

$$
\langle n(T)\rangle=A-B T .
$$

Equation (13) has a negative slope. The value of $B$ is around $10^{-4} \mathrm{~K}^{-1}$.

On the other hand, birefringence is dependent on the order parameter $S$. Based on Haller's approximation, the order parameter can be approximated as $S=\left(1-T / T_{c}\right)^{\beta}$. Thus, the temperature-dependent birefringence has the following form: ${ }^{18}$

$$
\Delta n(T)=(\Delta n)_{o}\left(1-T / T_{c}\right)^{\beta},
$$

In Eq. (14), $(\Delta n)_{o}$ is the LC birefringence in the crystalline state (or $T=0 \mathrm{~K}$ ), the exponent $\beta$ is a material constant, and $T_{c}$ is the clearing temperature of the LC material under investigation.

Substituting Eqs. (13) and (14) back to Eqs. (11) and (12), we derive the four-parameter model for describing the temperature effect on the LC refractive indices:

$$
\begin{aligned}
& n_{e}(T) \approx A-B T+\frac{2(\Delta n)_{o}}{3}\left(1-\frac{T}{T_{c}}\right)^{\beta}, \\
& n_{o}(T) \approx A-B T-\frac{(\Delta n)_{o}}{3}\left(1-\frac{T}{T_{c}}\right)^{\beta},
\end{aligned}
$$

At the first glance, Eqs. (15) and (16) seem to have four fitting parameters. As a matter of fact, parameters $A$ and $B$ can be obtained from fitting the temperature-dependent $\langle n(T)\rangle$ data at a given wavelength, and parameters $(\Delta n)_{o}$ and $\beta$ can be obtained by fitting the temperature-dependent $\Delta n$ data. Thus, once we have measured the temperaturedependent $n_{e}$ and $n_{o}$, we rearrange the data into $\langle n(T)\rangle$ and $\Delta n(T)$ and parameters $[A, B]$, and $\left[(\Delta n)_{o}, \beta\right]$ can be obtained fairly straightforwardly.

For direct-view and projection LC displays, the LC mixture employed usually exhibits a low birefringence and high clearing temperature $\left(T_{c}>90^{\circ} \mathrm{C}\right)$. Under the condition that $T \ll T_{c}$, the $\left(1-T / T_{c}\right)^{\beta}$ term in Eqs. (15) and (16) can be expanded into a power series. Keeping the first three terms, we obtain

$$
\begin{aligned}
& n_{e}(T)=A_{e}-B_{e} T-C_{e} T^{2}, \\
& n_{o}(T)=A_{o}-B_{o} T+C_{o} T^{2},
\end{aligned}
$$

where $\quad A_{e}=A+2(\Delta n)_{o} / 3, \quad B_{e}=B+2(\Delta n)_{o} \beta / 3 T_{c}$, $C_{e}=(\Delta n)_{o} \beta(1-\beta) / 3 T_{c}^{2}, \quad A_{o}=A-(\Delta n)_{o} / 3$, $B_{o}=B-(\Delta n)_{o} \beta / 3 T_{c}$, and $C_{o}=(\Delta n)_{o} \beta(1-\beta) / 6 T_{c}^{2}$. Equations (17) and (18) indicate that the LC refractive indices have a parabolic relationship with temperature for the lowbirefringence and high-clearing-point LC material when the operating temperature is far from the clearing point. For $n_{e}$, the placket of the parabola is downward, whereas for $n_{o}$, the placket is upward.

\section{EXPERIMENT}

We measured the refractive indices of 4-cyano-4-npentylbiphenyl (5CB), 4-cyano-4-n-pentyl-cyclohexanephenyl $(5 \mathrm{PCH})$, a high birefringence LC mixture (UCF-35; $\Delta n \sim 0.35$ ) formulated in our laboratory, and a commercial low- $\Delta n$ TFT LC mixture (Merck MLC-6241-000; $\Delta n$ $\left.\sim 0.085 ; T_{c}=373.72 \mathrm{~K}\right)$ using a multiwavelength Abbe refractometer (Atago DR-M4) at $\lambda=450,486,546,589,633$, and $656 \mathrm{~nm}$. For the LC samples studied, their $n_{e}$ or $n_{o}$ is outside the measurable range of the Abbe refractometer at $\lambda=450$ and $486 \mathrm{~nm}$. Thus, we will have fewer experimental data for these two wavelengths. The accuracy of the Abbe refractometer is up to the fourth decimal. For a given wavelength, we measured the refractive indices of $5 \mathrm{CB}, 5 \mathrm{PCH}$, MLC-6241-000 and UCF-35 from 10 to $60^{\circ} \mathrm{C}$. The temperature of the Abbe refractometer is controlled by a circulating constant temperature bath (Atago Model 60-C3). 


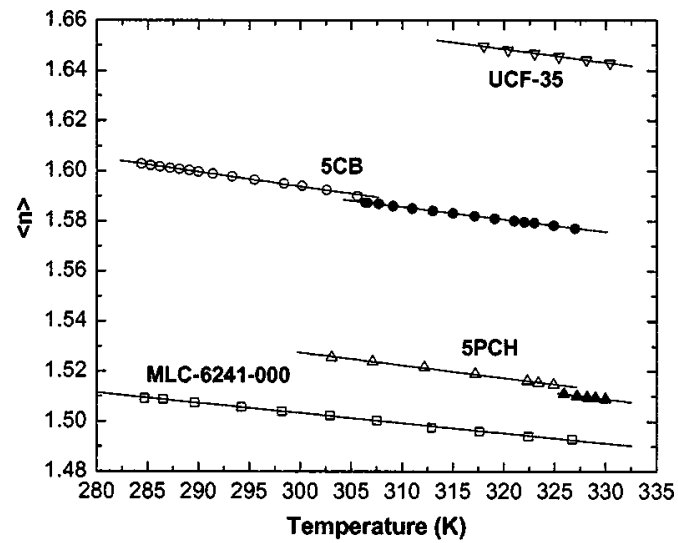

FIG. 1. Temperature-dependent average refractive index $\langle n\rangle$ of 5CB, 5PCH, MLC-6241-000, and UCF-35 at $\lambda=589 \mathrm{~nm}$. Open circles, upward-triangles, squares, and downward-triangles are average refractive indexes $\langle n\rangle$ of 5CB, 5PCH, MLC-6241-000, and UCF-35, respectively. Solid lines are fittings by using Eq. (13). The fitting parameters are listed in Table I. The filled circles and triangles are isotropic refractive indices for $5 \mathrm{CB}$ and $5 \mathrm{PCH}$, respectively.

To validate the four-parameter model, we use the model to fit the experimental data of 5CB, 5PCH, MLC-6241-000, and UCF-35 measured at $\lambda=546,589$, and $633 \mathrm{~nm}$. Excellent agreement between the four-parameter model and the experimental data is obtained. To validate the threeparameter parabolic model, we fit the model with the experimental data of MLC-6241-000. The agreement is also excellent.

\section{RESULTS AND DISCUSSION}

\section{A. Validation of the four-parameter model}

Although the four-parameter model has four unknowns, $A, B,(\Delta n)_{o}$, and $\beta$, they can be obtained by the two-step fittings. In experiment, we measured the refractive indices ( $n_{e}$ and $n_{o}$ ) using Abbe refractometer at six wavelengths in the temperature range from 10 to $60{ }^{\circ} \mathrm{C}$. Once the refractive indices are measured, birefringence and average refractive index can be calculated. The first step is to use Eq. (13) to fit the average refractive index data and obtain parameters $A$ and $B$. The second step is to use Eq. (14) to fit the birefringence data and extract $(\Delta n)_{o}$ and $\beta$. Finally, we plug these two sets of parameters into Eqs. (15) and (16) to calculate the refractive indices and compare the calculated results with the measured ones. We use four different materials: $5 \mathrm{CB}, 5 \mathrm{PCH}$, MLC-6241-000, and UCF-35 to validate the four-parameter model. At room temperature, the birefringences of UCF-35,

TABLE I. Fitting parameters for the average refractive index $\langle n\rangle$ and birefringence $(\Delta n)$ of four LCs at $\lambda=589 \mathrm{~nm}$.

\begin{tabular}{ccccccc}
\hline \hline & \multicolumn{2}{c}{$\langle n\rangle$} & & \multicolumn{2}{c}{$\Delta n$} \\
\cline { 2 - 3 } \cline { 5 - 6 } LC & $A$ & $B\left(\mathrm{~K}^{-1}\right)$ & & $(\Delta n)_{o}$ & $\beta$ \\
\hline 5CB & 1.7674 & $5.79 \times 10^{-4}$ & & 0.3505 & 0.1889 \\
5 PCH & 1.6795 & $5.07 \times 10^{-4}$ & & 0.1703 & 0.1512 \\
MLC-6241-000 & 1.6233 & $4.00 \times 10^{-4}$ & & 0.1224 & 0.2234 \\
UCF-35 & 1.8187 & $5.32 \times 10^{-4}$ & & 0.5727 & 0.2719 \\
\hline \hline
\end{tabular}

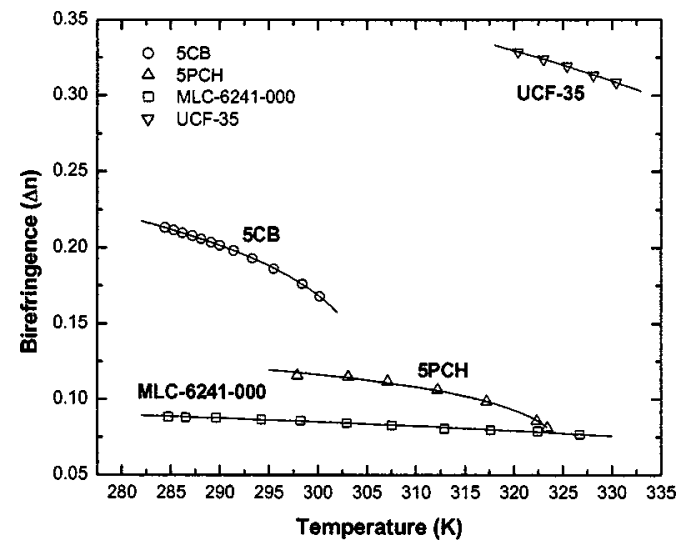

FIG. 2. Temperature-dependent birefringence $(\Delta n)$ of UCF-35 (open downward-triangles), $5 \mathrm{CB}$ (open circles), 5PCH (open upward-triangles), and MLC-6241-000 (open squares) at $\lambda=589 \mathrm{~nm}$. The four solid lines are fitting curves using $\Delta n=(\Delta n)_{o}\left(1-T / T_{c}\right)^{\beta}$, where $T_{c}$ is clearing point. The fitting parameters are listed on Table I.

5CB, 5PCH, and MLC-6241-000 are 0.35, 0.2, 0.12, and 0.085 , respectively, at $\lambda=589 \mathrm{~nm}$, and their clearing temperatures are $368.3,306.6,325.9$, and $373.7 \mathrm{~K}$, respectively. Excellent agreement is obtained between the calculated results and experimental data of these four different materials.

Figure 1 depicts the temperature-dependent average refractive indices of 5CB, 5PCH, UCF-35, and MLC-6241-000 at $\lambda=589 \mathrm{~nm}$. Circles, upward-triangles, squares, and downward-triangles represent experimental data for $5 \mathrm{CB}$, 5PCH, MLC-6241-000, and UCF-35, respectively, while solid lines are fitting results using Eq. (13). The fitting parameters for these four materials are listed in Table I. The average refractive index decreases linearly as the temperature increases. In the isotropic phase, the refractive index also decreases linearly with the increased temperature. Near the isotropic temperature, there is a sudden decrease in refractive index because of the second order phase transition which results in a density fluctuation. ${ }^{19}$ The filled circles and triangles in Fig. 1 are the isotropic refractive indices for 5CB and $5 \mathrm{PCH}$, respectively. They also decrease linearly with in-

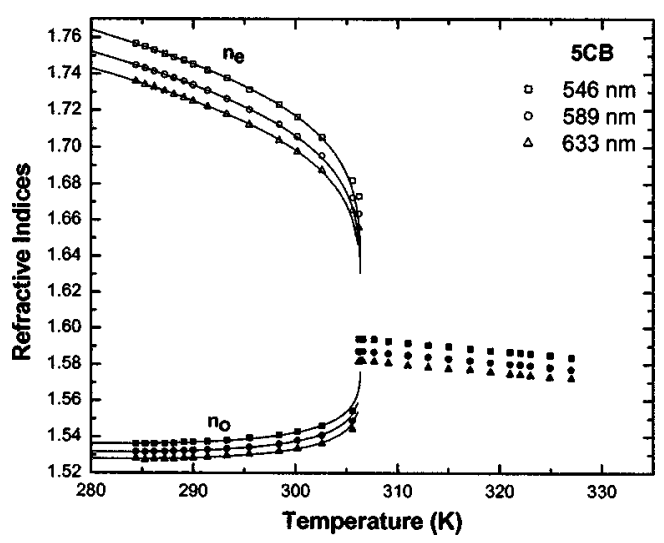

FIG. 3. Temperature-dependent refractive indices of $5 \mathrm{CB}$ at $\lambda=546,589$, and $633 \mathrm{~nm}$. Squares, circles, and triangles are experimental data for refractive indices measured at $\lambda=546,589$, and $633 \mathrm{~nm}$, respectively. Solid lines are fittings using the four-parameter model [Eqs. (15) and (16)]. The fitting parameters are listed in Table II. 
TABLE II. Fitting parameters for the average refractive index $\langle n\rangle$ and birefringence $(\Delta n)$ of $5 \mathrm{CB}$ at $\lambda=546,589$, and $633 \mathrm{~nm}$.

\begin{tabular}{ccccccc}
\hline \hline & \multicolumn{2}{c}{$\langle n\rangle$} & & \multicolumn{2}{c}{$\Delta n$} \\
\cline { 2 - 3 } \cline { 5 - 6 }$\lambda(\mathrm{nm})$ & $A$ & $B\left(\mathrm{~K}^{-1}\right)$ & & $(\Delta n)_{o}$ & $\beta$ \\
\hline 546 & 1.7751 & $5.81 \times 10^{-4}$ & & 0.3594 & 0.1862 \\
589 & 1.7674 & $5.79 \times 10^{-4}$ & & 0.3505 & 0.1889 \\
633 & 1.7601 & $5.75 \times 10^{-4}$ & & 0.3389 & 0.1855 \\
\hline \hline
\end{tabular}

creasing temperature in the isotropic state, but the slope is slightly different from that of the nematic state.

Figure 2 depicts the temperature-dependent birefringence of 5CB, 5PCH, MLC-6241-000, and UCF-35 at $\lambda$ $=589 \mathrm{~nm}$. Circles, upward-triangles, squares, and downward-triangles are experimental data for $5 \mathrm{CB}, 5 \mathrm{PCH}$, MLC-6241-000, and UCF-35, respectively, while solid lines are fitting results using Eq. (14). The fitting parameters for these four materials at $\lambda=589 \mathrm{~nm}$ are also listed in Table I. Through fittings, we obtain parameters $(\Delta n)_{o}$ and $\beta$.

For a full color display, we need to know the refractive indices at the three primary colors. Figure 3 depicts the temperature-dependent refractive indices of $5 \mathrm{CB}$ at $\lambda=546$, 589 , and $633 \mathrm{~nm}$. Squares, circles, and triangles are experimental data measured at $\lambda=546,589$, and $633 \mathrm{~nm}$, respectively, and solid lines are fitting results using the fourparameter model. The agreement is excellent. In an isotropic phase, the optical anisotropy disappears (i.e., $n_{e}=n_{o}$ ) regardless of wavelength. The fitting parameters for the fourparameter model are listed in Table II. As shown in Fig. 3, the agreement between the four-parameter model and experimental data is excellent.

Due to the shorter molecular conjugation, $5 \mathrm{PCH}$ has a lower birefringence than $5 \mathrm{CB}$, however, its clearing temperature is higher. Thus, similar to $5 \mathrm{CB}, 5 \mathrm{PCH}$ has been widely used in many commercial LC mixtures. Figure 4 depicts the temperature-dependent refractive indices of $5 \mathrm{PCH}$ at $\lambda$ $=546,589$, and $633 \mathrm{~nm}$. Squares, circles, and triangles are the experimental data measured at $\lambda=546,589$, and $633 \mathrm{~nm}$,

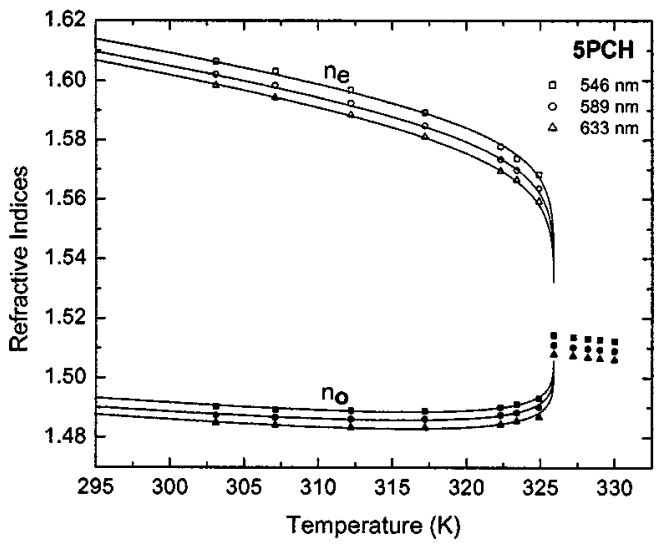

FIG. 4. Temperature-dependent refractive indices of $5 \mathrm{PCH}$ at $\lambda=546,589$, and $633 \mathrm{~nm}$. Squares, circles, and triangles are experimental data for refractive indices measured at $\lambda=546,589$, and $633 \mathrm{~nm}$, respectively. Solid lines are fittings using the four-parameter model [Eqs. (15) and (16)]. The fitting parameters are listed in Table III.
TABLE III. Fitting parameters for the average refractive index $\langle n\rangle$ and birefringence $(\Delta n)$ of $5 \mathrm{PCH}$ at $\lambda=546,589$, and $633 \mathrm{~nm}$.

\begin{tabular}{ccccccc}
\hline \hline & \multicolumn{2}{c}{$\langle n\rangle$} & & \multicolumn{2}{c}{$\Delta n$} \\
\cline { 2 - 3 } \cline { 5 - 6 }$\lambda(\mathrm{nm})$ & $A$ & \multirow{2}{*}{$B\left(\mathrm{~K}^{-1}\right)$} & & $(\Delta n)_{o}$ & $\beta$ \\
\hline 546 & 1.6837 & $5.09 \times 10^{-4}$ & & 0.1719 & 0.1473 \\
589 & 1.6795 & $5.06 \times 10^{-4}$ & & 0.1703 & 0.1491 \\
633 & 1.6684 & $4.82 \times 10^{-4}$ & & 0.1682 & 0.1487 \\
\hline \hline
\end{tabular}

respectively, and solid lines are fitting results using the fourparameter model. Above the clearing point $\left(T_{c}=325.9 \mathrm{~K}\right)$, the optical anisotropy vanishes so that $n_{e}=n_{o}$. The fitting parameters for the four-parameter model are listed in Table III. Excellent agreement between the four-parameter model and experimental data is achieved.

Figure 5 depicts the temperature-dependent refractive indices of MLC-6241-000 and UCF-35 at $\lambda=589 \mathrm{~nm}$. Circles and squares are measured refractive indices of MLC-6241000 and UCF-35, respectively. Solid lines are fitting results using the four-parameter model. The fitting parameters for the four-parameter model are listed in Table I. Excellent agreement is achieved between the four-parameter model and experimental data.

Summarizing, the four-parameter model fits very well with all the four LC materials we studied, although their birefringence values span a wide range. It is an accurate model to describe the temperature effect on the refractive indices of liquid crystals.

\section{B. Validation of the parabolic model}

For a low- $\Delta n$, high- $T_{c}$ LC compound or mixture, the four-parameter model can be reduced to a three-parameter parabolic model. To validate the parabolic model, we chose two LC mixtures: MLC-6241-000 $(\Delta n \sim 0.085)$ and UCF-35 $(\Delta n \sim 0.35)$; their clearing temperatures are 100 and $95.3{ }^{\circ} \mathrm{C}$, respectively. Figure 5 depicts the temperature-dependent re-

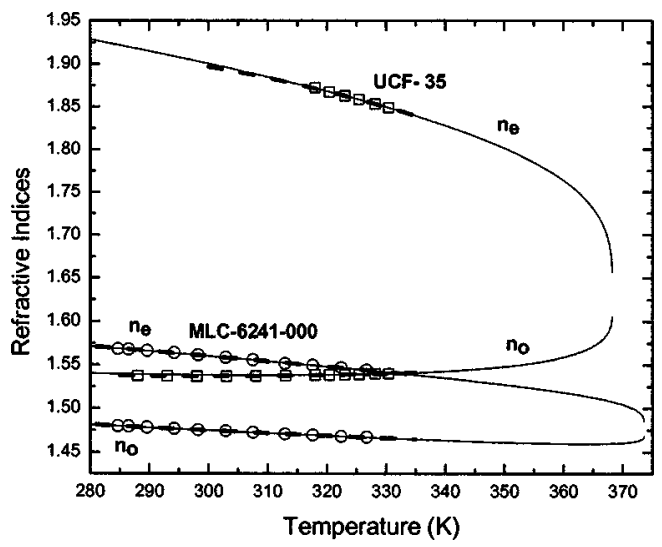

FIG. 5. Temperature-dependent refractive indices of UCF-35 and MLC6241-000 at $\lambda=589 \mathrm{~nm}$. Squares and circles are experimental data for refractive indices of UCF-35 and MLC-6241-000, respectively. Solid lines are fittings using the four-parameter model [Eqs. (15) and (16)]. Dashed lines are fittings using the three-parameter parabolic model [Eqs. (17) and (18)]. The fitting parameters for the two models are listed in Tables I and IV, respectively. 
TABLE IV. Fitting parameters for the three-parameter parabolic model [Eqs. (17) and (18)]. $\lambda=589 \mathrm{~nm}$. The LC mixtures studied are MLC-6241-000 and UCF-35.

\begin{tabular}{|c|c|c|c|c|c|c|}
\hline \multirow[b]{2}{*}{ Materials } & \multicolumn{3}{|c|}{$n_{e}$} & \multicolumn{3}{|c|}{$n_{o}$} \\
\hline & $A_{e}$ & $B_{e}\left(\mathrm{~K}^{-1}\right)$ & $C_{e}\left(\mathrm{~K}^{-2}\right)$ & $A_{o}$ & $B_{o}\left(\mathrm{~K}^{-1}\right)$ & $C_{o}\left(\mathrm{~K}^{-2}\right)$ \\
\hline MLC-6241-000 & 1.6495 & $2.00 \times 10^{-5}$ & $9.25 \times 10^{-7}$ & 1.6343 & $7.50 \times 10^{-4}$ & $7.21 \times 10^{-7}$ \\
\hline UCF-35 & 1.0367 & -0.0069 & $1.00 \times 10^{-5}$ & 1.8276 & 0.00194 & $3.24 \times 10^{-6}$ \\
\hline
\end{tabular}

fractive indices of MLC-6241-000 and UCF-35 at $\lambda$ $=589 \mathrm{~nm}$. Circles and squares are measured refractive indices of MLC-6241-000 and UCF-35, respectively. Solid lines are fitting results using the four-parameter model and dashed lines are fitting results using the three-parameter parabolic model. The fitting parameters for the three-parameter parabolic model are listed in Table IV. For MLC-6241-000, the fitting parameters $\left(A_{e}, B_{e}, C_{e}\right)$ for $n_{e}$ are $(1.6495,2.00$ $\left.\times 10^{-5}, 9.25 \times 10^{-7}\right)$ and $\left(A_{o}, B_{o}, C_{o}\right)$ for $n_{o}$ are $\left(1.6343,7.50 \times 10^{-4}, 7.21 \times 10^{-7}\right)$, respectively. For UCF35 , the fitting parameters $\left(A_{e}, B_{e}, C_{e}\right)$ for $n_{e}$ are (1.0367, $\left.-0.0069,1.00 \times 10^{-5}\right)$ and $\left(A_{o}, B_{o}, C_{o}\right)$ for $n_{o}$ are $\left(1.8276,0.0019,3.24 \times 10^{-6}\right)$, respectively.

At room temperature, the birefringences of MLC-6241000 and UCF-35 are around 0.085 and 0.35 at $\lambda=589 \mathrm{~nm}$, respectively. In Fig. 5, the three-parameter parabolic model (dashed lines) fits the experimental data of the two materials very well and almost overlap each other with the fourparameter model when the temperature is far from the clearing point. In the three-parameter model, we predict all the $A_{e, o}, B_{e, o}$ and $C_{e, o}$ coefficients should be positive. From this viewpoint, the three-parameter parabolic model fits the experimental data of MLC-6241-000 better than that of UCF-35 because one of the fitting parameters $B_{e}$ for UCF-35 is negative although its value is relatively small. To explain this discrepancy, we need to review the assumption made during the derivation of Eqs. (17) and (18). The two assumptions made there are: (1) the LC birefringence is low, and (2) $T \ll T_{c}$. Under these conditions, the four-parameter model can be expanded into a power series, which leads to the

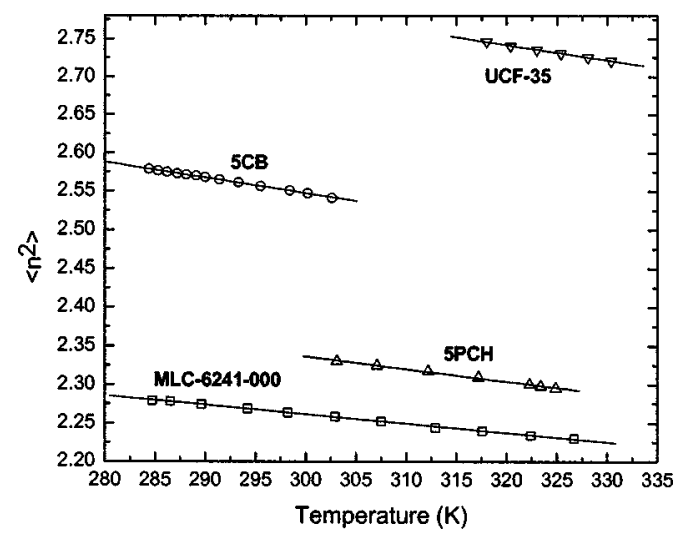

FIG. 6. Temperature-dependent average squared refractive indices $\left\langle n^{2}\right\rangle$ of $5 \mathrm{CB}$, 5PCH, MLC-6241-000, and UCF-35 at $\lambda=589 \mathrm{~nm}$. Open circles, upward-triangles, squares, and downward-triangles are average squared refractive indices $\left\langle n^{2}\right\rangle$ of 5CB, 5PCH, MLC-6241-000, and UCF-35, respectively. Solid lines are fittings by using Eq. (22). The fitting parameters are listed in Table V. three-parameter parabolic model. However, the birefringence of UCF-35 is quite high, so that the power expansion is less accurate. Consequently, the fitting results for UCF-35 are less satisfactory. Thus, the three-parameter parabolic model is more suitable for the low-birefringence LC materials while the operating temperature is far from the clearing temperature.

\section{Temperature effect on $\left\langle n^{2}\right\rangle$}

From Eq. (2), $\left\langle n^{2}\right\rangle=\left(n_{e}^{2}+2 n_{o}^{2}\right) / 3$. Based on the Vuks equation, we derived a relationship between $\langle n\rangle$ and $\left\langle n^{2}\right\rangle$ :

$$
\frac{\langle n\rangle}{\left\langle n^{2}\right\rangle+2} \approx \frac{\sqrt{2}}{4} .
$$

Equation (19) can be rearranged as

$$
\left\langle n^{2}\right\rangle \approx 2 \sqrt{2}\langle n\rangle-2 \text {. }
$$

Substituting Eq. (13) into Eq. (20), we derive the following equation:

$$
\left\langle n^{2}\right\rangle \approx(2 \sqrt{2} A-2)-2 \sqrt{2} B T .
$$

Letting $C=(2 \sqrt{2} A-2)$ and $D=2 \sqrt{2} B$, we rewrite

$$
\left\langle n^{2}(T)\right\rangle \approx C-D T \text {. }
$$

Equation (22) predicts that $\left\langle n^{2}\right\rangle$ decreases linearly as the temperature increases.

Figure 6 depicts the temperature-dependent $\left\langle n^{2}\right\rangle$ of 5CB, $5 \mathrm{PCH}$, UCF-35, and MLC-6241-000 measured at $\lambda$ $=589 \mathrm{~nm}$. The open circles, upward-triangles, squares, and downward-triangles are the experimental data of $\left\langle n^{2}\right\rangle$ for 5CB, 5PCH, MLC-6241-000, and UCF-35, respectively. The solid lines are fittings results using Eq. (22). The fitting parameters are listed in Table V. Similarly to $\langle n\rangle,\left\langle n^{2}\right\rangle$ decreases linearly as the temperature increases. The difference is that $\left\langle n^{2}\right\rangle$ decreases faster than $\langle n\rangle$. Comparing Eq. (22) with Eq. (13), the slope of Eq. (22) is larger than that of Eq. (13). From the fitting results, indeed, the slope of $\left\langle n^{2}\right\rangle$ is $\sim 3$

\begin{tabular}{|c|c|c|c|c|}
\hline \multirow[b]{2}{*}{ Coefficients } & \multicolumn{4}{|c|}{ Materials } \\
\hline & $5 \mathrm{CB}$ & $5 \mathrm{PCH}$ & MLC-6241-000 & UCF-35 \\
\hline C & 3.1603 & 2.8218 & 2.6250 & 3.3958 \\
\hline$D\left(\mathrm{~K}^{-1}\right)$ & $2.04 \times 10^{-3}$ & $1.62 \times 10^{-3}$ & $1.21 \times 10^{-3}$ & $2.04 \times 10^{-3}$ \\
\hline
\end{tabular}
times larger than that of $\langle n\rangle$.

TABLE V. Fitting parameters for average squared refractive index $\left\langle n^{2}\right\rangle$. LC: $5 \mathrm{CB}, 5 \mathrm{PCH}, \mathrm{MLC}-6241-000$ and UCF-35 at $\lambda=589 \mathrm{~nm}$. 


\section{CONCLUSIONS}

We have derived a four-parameter model for describing the temperature effect on the refractive indices of liquid crystals based on the Vuks equation. Four different LC materials with different birefringences are used to validate this fourparameter model. Excellent agreement between the experimental data and theory is obtained. For a TFT LC material with low birefringence and high clearing point, the fourparameter model can be simplified to the three-parameter parabolic model. The three-parameter parabolic model fits well with the experimental data of MLC-6241-000, which is a low-birefringence and high-clearing-temperature LC mixture. The three-parameter parabolic model fits the experimental data very well when the temperature is far from the clearing point. Thus, the three-parameter parabolic model is more suitable for describing the temperature effect on TFT LC materials with a low birefringence and a high clearing point. Finally, we find that $\left\langle n^{2}\right\rangle$ also decreases linearly as the temperature increases.

\section{ACKNOWLEDGMENTS}

The authors are indebted to the financial support of AFOSR under Contract No. F49620-01-1-0377.
${ }^{1}$ E. Lueder, Liquid Crystal Displays (Wiley, New York, 2001).

${ }^{2}$ E. H. Stupp and M. S. Brennesholtz, Projection Displays (Wiley, New York, 1998)

${ }^{3}$ S. T. Wu and D. K. Yang, Reflective Liquid Crystal Displays (Wiley, New York, 2001).

${ }^{4}$ I. C. Khoo and S. T. Wu, Optics and Nonlinear Optics of Liquid Crystals (World Scientific, Singapore, 1993).

${ }^{5}$ S. T. Wu, Phys. Rev. A 33, 1270 (1986).

${ }^{6}$ S. T. Wu, J. Appl. Phys. 69, 2080 (1991).

${ }^{7}$ I. Abdulhalim, Mol. Cryst. Liq. Cryst. 197, 103 (1991).

${ }^{8}$ S. T. Wu, C. S. Wu, M. Warenghem, and M. Ismaili, Opt. Eng. 32, 1775 (1993).

${ }^{9}$ E. M. Averyanov, J. Opt. Technol. 64, 417 (1997).

${ }^{10}$ J. Li and S. T. Wu, J. Appl. Phys. 95, 896 (2004).

${ }^{11}$ J. Li, S. Gauza, and S. T. Wu, Opt. Express 12, 2002 (2004).

${ }^{12}$ W. H. de Jeu, Physical Properties of Liquid Crystalline Materials (Gordon and Breach, New York, 1980), Chap. 4.

${ }^{13}$ I. C. Khoo and R. Normandin, IEEE J. Quantum Electron. QE-21, 329 (1985).

${ }^{14}$ M. Warenghem and G. Joly, Mol. Cryst. Liq. Cryst. 197, 103 (1991).

${ }^{15}$ M. F. Vuks, Opt. Spektrosk. 20, 644 (1966).

${ }^{16}$ For example, see J. D. Jackson, Classical Electrodynamics, 2nd Ed. (Wiley, New York, 1962).

${ }^{17}$ L. M. Blinov, Electro-Optical and Magneto-Optical Properties of Liquid Crystals (Wiley, New York, 1983), Chap. 2.

${ }^{18}$ I. Haller, Prog. Solid State Chem. 10, 103 (1975).

${ }^{19}$ H. R. Zeller, Phys. Rev. A 26, 1785 (1982). 